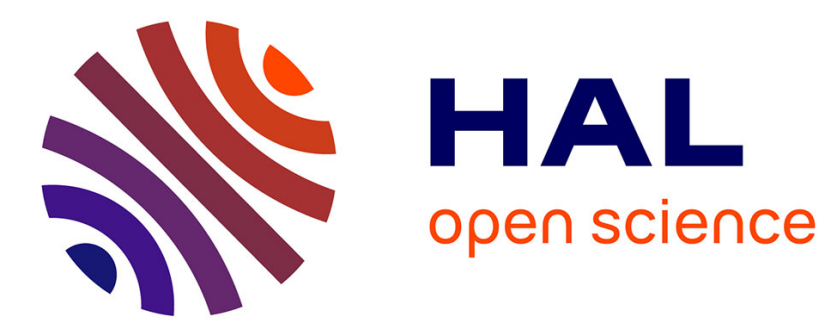

\title{
On parameter tuning and convergence properties of the DREM procedure
}

Marina Korotina, Stanislav Aranovskiy, Rosane Ushirobira, Alexey Vedyakov

\section{To cite this version:}

Marina Korotina, Stanislav Aranovskiy, Rosane Ushirobira, Alexey Vedyakov. On parameter tuning and convergence properties of the DREM procedure. ECC 2020 - 18th European Control Conference, May 2020, Saint Petersburg, Russia. 10.23919/ECC51009.2020.9143808 . hal-02523112

\section{HAL Id: hal-02523112 \\ https://hal.inria.fr/hal-02523112}

Submitted on 28 Mar 2020

HAL is a multi-disciplinary open access archive for the deposit and dissemination of scientific research documents, whether they are published or not. The documents may come from teaching and research institutions in France or abroad, or from public or private research centers.
L'archive ouverte pluridisciplinaire HAL, est destinée au dépôt et à la diffusion de documents scientifiques de niveau recherche, publiés ou non, émanant des établissements d'enseignement et de recherche français ou étrangers, des laboratoires publics ou privés. 


\title{
On parameter tuning and convergence properties of the DREM procedure
}

\author{
Marina Korotina $^{1}$, Stanislav Aranovskiy ${ }^{2,1}$, Rosane Ushirobira ${ }^{3}$, Alexey Vedyakov ${ }^{1}$
}

\begin{abstract}
The recently proposed Dynamic Regressor Extension and Mixing (DREM) procedure has been proven to enhance transient performance in online parameter estimation and it has been successfully applied to a variety of adaptive control problems and applications. However, to use this procedure, a linear operator has to be chosen to perform the dynamic extension. A poor choice of the operator can reduce excitation of signals and hence it can compromise convergence properties. This paper presents a systematic selection of operators such that the excitation is always preserved. The paper also studies convergence conditions when the DREM procedure is combined with a least-squares estimator.
\end{abstract}

\section{INTRODUCTION}

The linear regression equation (LRE) plays a central role in adaptive parameter estimation and adaptive control. It appears in system identification [1], in model-reference adaptive control [2], [3] and adaptive pole-placement [4], in filtering and prediction [5], in reinforcement learning [6], and in many others areas. The linear regression model is given by

$$
y(t)=\phi^{\top}(t) \theta+w(t),
$$

where $y(t) \in \mathbb{R}$ is the output signal, $\phi(t) \in \mathbb{R}^{n}$ is the regressor, $w(t) \in \mathbb{R}$ is an additive distortion, e.g., a measurement noise, and $\theta \in \mathbb{R}^{n}$ is the vector of unknown constant parameters. The signals $y$ and $\phi$ are known, e.g., measured, and the distortion signal $w$ is unknown. The goal is to estimate the vector of parameters $\theta$ using the measurements $y$ and $\phi$. The model (1) is an LRE with a scalar output, but a more general case of $y(t) \in \mathbb{R}^{p}$ and $\phi(t) \in \mathbb{R}^{n \times p}$ can be also considered.

Two classical approaches can be deployed to the parameter estimation problem: the gradient and the least-squares estimators. Several modifications of these approaches are known, e.g., the normalized gradient estimator, the leastsquares estimator with forgetting factor, and others, see [2], [3]. For both methods, the convergence of the estimates is based on the key assumption of sufficient and uniform (in time) informational richness of the regressor $\phi$, which is rigorously defined as the persistence of excitation (PE) condition, see Section II for the definition. The drawback

\footnotetext{
*This work was supported by the Ministry of Science and Higher Education of Russian Federation, passport of goszadanie no. 2019-0898, and by Rennes Métropole (France), project AIS19C0326. The corresponding author is S. Aranovskiy, contact email: stanislav.aranovskiy@centralesupelec.fr.

${ }^{1}$ Faculty of Control Systems and Robotics, ITMO University, 197101 Saint Petersburg, Russia.

${ }^{2}$ IETR - CentaleSupélec, Avenue de la Boulaie, 35576 Cesson-Sévigné, France.

${ }^{3}$ Inria, Univ. Lille, CNRS, UMR 9189 - CRIStAL, F-59000 Lille, France.
}

of these techniques is that whereas the weak monotonicity of a (weighted) norm of estimation errors is guaranteed, the element-wise estimation transients for each component of the vector $\theta$ are hardly predictable and typically exhibit significant oscillations and peaking phenomena. Moreover, tuning procedures for these estimators usually involve the tuning of a gain matrix and they can be somewhat unclear requiring multiple trial-and-error attempts. Notably, it has been shown in [7] that for gradient estimators, the amplification of the gains does not always yield accelerated transients, but it increases the peaking.

The Dynamic Regressor Extension and Mixing (DREM) procedure has been recently proposed in [8]. In [9], the authors propose an interpretation of this procedure as a functional Luenberger observer. The DREM approach has been successfully applied to a variety of adaptive control problems and applications, such as direct model reference control [10], time-varying frequency estimation [11], electrical drives velocity estimation [12], and power system applications [13], [14]. Among many, the DREM procedure has the following advantages:

- it provides a novel convergence condition that differs from the PE requirement, and it is less restrictive in certain scenarios;

- independently of the excitation conditions, it ensures the element-wise transient monotonicity precluding oscillations and peaking;

- each element of the estimate of $\theta$ is tuned with a separate scalar gain, which does not affect transients for other elements; this property makes the gain tuning simple and transparent.

Two basic steps are involved in the DREM procedure, namely the dynamic regressor extension (DRE) and mixing steps. At the first step, a linear dynamic operator is introduced to extend the original linear regression equation (1) and to obtain an extended matrix regressor. At the second step, a nonlinear transformation is applied to the previously generated data to obtain a set of $n$ scalar independent LREs for each component of the vector $\theta$ sharing the same new scalar regressor. Finally, the gradient estimator is applied to each of these scalar equations.

The key question of the DREM procedure is the choice of the linear operator for dynamics extension at the first step. As it has been shown in [8], a bad choice of this operator can compromise the convergence even if the original regressor $\phi$ is PE. In the discrete-time domain, a summation over a fixed window has been proposed in [15]; however, to choose 
the window size, a certain a priori knowledge about the original regressor $\phi$ must be available. This problem has also been considered in [16] for a particular class of LRE, where the regressor consists of a finite sum of sinusoidal signals and the upper frequency bound is known. Motivated by the work [17], the authors in [18] and [15] have also proposed to use the Kreisselmeier's regressor extension, where the authors of [18] have used the name memory regressor extension. However, these papers do not address properties and applicability conditions of such a choice, and the operator design for the DRE step of the DREM procedure remains an open question.

The use of the gradient estimator in the DREM procedure allows establishing the convergence under the non-squareintegrability condition of the new scalar regressor, which differs from the PE condition. An open question is whether the use of the least-squares estimator (LSQ) instead of the gradient estimator will preserve convergence under the same conditions or it will yield different conditions.

Novelty and contribution. The contribution of this paper is twofold. First, we consider the Kreisselmeier's regressor extension in the context of the DREM procedure and we study its properties and applicability conditions. We show that this choice does not require any prior knowledge about the original regressor and that the PE property is always preserved. Moreover, we also analyze how the excitation propagates through the proposed dynamics. Second, we consider the DREM procedure when the gradient estimator is replaced with the least-squares estimator, with or without forgetting modification; we compare necessary and sufficient convergence conditions in the context of the DREM procedure.

Results of this paper are presented for continuous time only; however, they can be extended to the discrete time domain in a straightforward way.

The rest of this paper is organized as follows. For the completeness of the presentation, a brief description of the DREM procedure and some background material are given in Section II. The Kreisselmeier's regressor extension is studied in Section III, and the LSQ estimator in the context of the DREM procedure is considered in Section IV. Illustrative simulations are presented in Section $\mathrm{V}$, and the conclusive remarks are in Section VI.

Notation. For a positive integer $n, I_{n}$ denotes the $n \times n$ identity matrix. For a signal of time $x: \mathbb{R}_{+} \rightarrow \mathbb{R}$ and a linear operator $\mathcal{H}$, we denote the action of this operator on the signal $x$ as $\mathcal{H}[x]$. For a function $x: \mathbb{R}_{+} \rightarrow \mathbb{R}$, we say that $x \in \mathcal{L}_{2}$ if $\int_{0}^{t} x^{2}(s) d s$ converges to a constant as $t$ tends to $+\infty$. If the integral does not converge, we write $x \notin \mathcal{L}_{2}$.

\section{BACKGROUND MATERIAL}

The persitence of excitation $(P E)$ : First, we present the definition of the $(T, \mu)$-PE property.

Definition 1: A bounded signal $\phi: \mathbb{R}_{+} \rightarrow \mathbb{R}^{n}$ is $(T, \mu)$ persistently exciting if there exist scalars $T>0$ and $\mu>0$ such that for all $t \in \mathbb{R}_{+}$,

$$
\int_{t}^{t+T} \phi(s) \phi^{\top}(s) d s \geq \mu I_{n} .
$$

This property is further denoted as $\phi \in \mathrm{PE}$, or $\phi$ is PE.

The gradient estimators [2].: For all $t \in \mathbb{R}_{+}$, the gradient estimator for the LRE (1) is given by

$$
\dot{\hat{\theta}}(t)=\Gamma \phi(t)\left(y(t)-\phi^{\top}(t) \hat{\theta}(t)\right),
$$

where $\hat{\theta}$ denotes the estimate of $\theta$ and $\Gamma>0$ is the gain matrix. Define the estimation error $\tilde{\theta}(t)=\hat{\theta}(t)-\theta, \forall t \in \mathbb{R}_{+}$. Then the error dynamics is given by

$$
\dot{\tilde{\theta}}(t)=-\Gamma \phi(t) \phi^{\top}(t) \tilde{\theta}(t)+\Gamma \phi(t) w(t), \forall t \in \mathbb{R}_{+} .
$$

In the noise-free scenario, i.e. assuming $w \equiv 0$, the gradient estimator ensures exponential convergence to zero of the error $\tilde{\theta}$ if and only if the regressor $\phi$ is PE. In this case the gradient estimator is also input-to-state stable with respect to the noise $w$. Some conditions that are sufficient, and some conditions that are necessary for asymptotic convergence when $\phi$ is not PE have been discussed in [19]; however, they are very technical and can be hardly applied in practice.

The DREM procedure: To apply the DREM procedure, we start by performing the dynamic regressor extension step. For that, we introduce a linear, single-input $n$-output, boundedinput bounded-output (BIBO)-stable operator $\mathcal{H}$ and define the vector $Y: \mathbb{R}_{+} \rightarrow \mathbb{R}^{n}$ and the matrix $\Phi: \mathbb{R}_{+} \rightarrow \mathbb{R}^{n \times n}$ by:

$$
Y=\mathcal{H}[y], \Phi=\mathcal{H}\left[\phi^{\top}\right] .
$$

Due to the linearity of the operator $\mathcal{H}$ and BIBO stability, these signals satisfy

$$
Y(t)=\Phi(t) \theta+W(t), \forall t \in \mathbb{R}_{+},
$$

where $W=\mathcal{H}[w]$. For example, the operator $\mathcal{H}$ can produce an LTI system or can be chosen as a delay operator, as proposed in [8].

Next, a mixing step is applied to obtain a set of $n$ scalar equations. Recall that for any square and possibly singular $n \times n$ matrix $A$, we have $\operatorname{adj}(A) A=\operatorname{det}(A) I_{n}$, where $\operatorname{adj}(\cdot)$ is the adjoint (also called adjugate) matrix. Multiplying (3) by adj $(\Phi(t))$ from the left, we get

$$
\mathcal{Y}_{i}(t)=\Delta(t) \theta_{i}+\mathcal{W}_{i}(t)
$$

where $i \in \bar{n}=\{1,2, \ldots, n\}$, the scalar function $\Delta: \mathbb{R}_{+} \rightarrow$ $\mathbb{R}$ is defined as

$$
\Delta(t)=\operatorname{det} \Phi(t), \forall t \in \mathbb{R}_{+}
$$

and $\mathcal{Y}(t)=\operatorname{adj}(\Phi(t)) Y(t), \mathcal{W}(t)=\operatorname{adj}(\Phi(t)) W(t), \forall t \in$ $\mathbb{R}_{+}$. It is worth noting that for a bounded regressor $\phi$, the vector $\mathcal{W}$ is also bounded, and $w \equiv 0$ implies $\mathcal{W} \equiv 0$.

The set of $n$ scalar LRE (4) sharing the same bounded scalar regressor $\Delta$ is the result of the DREM procedure. Applying then the gradient estimator to (4) as

$$
\dot{\hat{\theta}}_{i}(t)=\gamma_{i} \Delta(t)\left(\mathcal{Y}_{i}(t)-\Delta(t) \hat{\theta}_{i}(t)\right),
$$


where $\gamma_{i}>0$ is a scalar tuning parameter, we obtain

$$
\dot{\tilde{\theta}}_{i}(t)=-\gamma_{i} \Delta^{2}(t) \tilde{\theta}_{i}(t)+\gamma_{i} \Delta(t) \mathcal{W}_{i}(t) .
$$

Assuming the noise-free scenario $w \equiv 0$, the following properties hold:

P1: $\Delta \notin \mathcal{L}_{2} \Leftrightarrow|\tilde{\theta}| \rightarrow 0$ asymptotically;

$\mathrm{P} 2: \Delta$ is $\mathrm{PE} \Leftrightarrow|\tilde{\theta}| \rightarrow 0$ exponentially fast;

P3: (element-wise monotonicity) for all $i \in \bar{n}$ for $t_{a} \leq t_{b}$ it holds $\left|\tilde{\theta}_{i}\left(t_{a}\right)\right| \leq\left|\tilde{\theta}_{i}\left(t_{b}\right)\right|$

P4: (element-wise tuning) variations in the gain $\gamma_{i}$ affect the transients for $\hat{\theta}_{i}$ only.

Concerning the case $w \not \equiv 0$, the estimator (6) is input-tostate stable with respect to $\mathcal{W}_{i}$ if $\Delta \in \mathrm{PE}$, which is a similar result as for the standard gradient estimator discussed above. Moreover, as it has been shown in [20], if $\mathcal{W}_{i} \in \mathcal{L}_{2}$ and $\Delta \notin \mathcal{L}_{2}$, then $\tilde{\theta}_{i}$ is bounded.

The property P1 illustrates the new convergence condition, namely the non-square-integrability of $\Delta$. As shown in [8], this condition is weaker than $\mathrm{PE}$ for $\phi$, where the price paid is the asymptotic convergence instead of the exponential one. To get the exponential convergence with the DREM procedure, the PE property of $\Delta$ must be satisfied. Therefore, the main design question when applying the DREM procedure is to choose an operator $\mathcal{H}$ such that the PE property of the original regressor $\phi$ is preserved. Such a choice is discussed in the following section.

Remark 1: Let us remark that the computation of the adjoint matrix adj $(\Phi)$ can be avoided in numerical implementations of the DREM estimator. So the elements $\mathcal{Y}_{i}$ in (4) can be computed using the Cramer's rule as

$$
\mathcal{Y}_{i}(t)=\operatorname{det} \Phi_{Y, i}(t), \forall t \in \mathbb{R}_{+},
$$

where $\Phi_{Y, i}$ is the matrix $\Phi$ where the $i$-th column is replaced with the vector $Y$, and $i \in \bar{n}$.

\section{THE EXCITATION-PRESERVING REGRESSOR EXTENSION}

\section{A. Excitation propagation}

Let the operator $\mathcal{H}$ be chosen such that the relationship $y_{u}=\mathcal{H}[u]$ for an input signal $u(t) \in \mathbb{R}$ and an output signal $y_{u}(t) \in \mathbb{R}^{n}$ have the following linear time-varying state-space representation:

$$
\begin{aligned}
\dot{x}(t) & =-a x(t)+\phi(t) u(t), \\
y_{u}(t) & =x(t),
\end{aligned}
$$

where $x(t) \in \mathbb{R}^{n}$ is the internal state vector, and $a>0$ is the tuning parameter. Then $\Phi=\mathcal{H}\left[\phi^{\top}\right]$ and $Y=\mathcal{H}[y]$ yield the Kreisselmeier's regressor extension (see [15]) and can be found as solutions of

$$
\begin{aligned}
& \dot{\Phi}(t)=-a \Phi(t)+\phi(t) \phi^{\top}(t), \\
& \dot{Y}(t)=-a Y(t)+\phi(t) y(t)
\end{aligned}
$$

for some initial values $\Phi(0)=\Phi_{0} \geq 0$ and $Y(0)=Y_{0}$.

The regressor extension (7), (8) is widely used in adaptive control. Particularly, it was used in [17] and recently in [21],
[22] to obtain the matrix equation $Y(t)=\Phi(t) \theta, \forall t \in \mathbb{R}_{+}$. Also in [18] the authors have applied a similar approach under the name memory regressor extension. However, to the best of our knowledge, the applicability conditions of (7), (8) for the DREM procedure and the PE property of the determinant of the matrix $\Phi$ have not been previously studied. To this end, we present the following theorem showing that (7) preserves the persistence of excitation.

Theorem 1: Let the signal $\phi: \mathbb{R}_{+} \rightarrow \mathbb{R}^{n}$ be $(T, \mu)-\mathrm{PE}$ and let $\Phi: \mathbb{R}_{+} \rightarrow \mathbb{R}^{n \times n}$ be a solution of (7). Then the following implication holds

$$
\phi \in \mathrm{PE} \Rightarrow \Delta \in \mathrm{PE},
$$

where $\Delta: \mathbb{R}_{+} \rightarrow \mathbb{R}$ is the determinant of $\Phi$. More precisely, for any positive integer $q \geq 1$ and for all $t \geq q T$, it holds

$$
\Delta(t) \geq \mu^{n}\left(\sum_{k=1}^{q} e^{-a k T}\right)^{n}
$$

and

$$
\liminf _{t \rightarrow \infty} \Delta(t) \geq\left(\frac{\mu}{e^{a T}-1}\right)^{n} .
$$

The proof Theorem is omitted due to the lack of space.

Remark 2: Theorem 1 motivates the use of (7), (8) as a reasonable choice for the dynamic regressor extension step of the DREM procedure. Under this choice, the PE property of the original regressor is always preserved and no prior knowledge about the regressor $\phi$ (e.g., the excitation period length $T$ ) is required. Moreover, after the first excitation interval, the new regressor $\Delta$ remains positive for all $t$, where the lower asymptotic bound on $\Delta$ can be computed as (10).

\section{B. Dynamics of the regressor $\Delta$}

Theorem 1 provides the lower bounds (9), (10) for the dynamic regressor extension (7). However, it is also possible to derive the dynamics equation of the new regressor signal $\Delta$. Such a result provides bounds on the time derivative of the regressor $\Delta$ and allows the estimation of the rate of its variation as it is stated in the following Proposition.

Proposition 1: Let $\Phi$ be a solution of (7) and let $\lambda_{M}$ denote the maximum eigenvalue of $\Phi$ and $\Delta=\operatorname{det} \Phi$. Then:

- if $\lambda_{M}=0$, then $\dot{\Delta}(t)=0$;

- if $\lambda_{M}>0$, then

$$
\dot{\Delta}(t) \geq\left(-a n+\frac{\|\phi(t)\|^{2}}{\lambda_{M}(t)}\right) \Delta(t), \forall t \in \mathbb{R}_{+} .
$$

The proof of Proposition is omitted due to the lack of space.

It is also worth noting that an upper bound of the maximum eigenvalue $\lambda_{M}$ can be estimated given an upper bound of $\phi$.

\section{THE LSQ ESTIMATION IN THE DREM PROCEDURE}

In the noise-free scenario, the gradient estimator (6) ensures exponential convergence when $\Delta \in \mathrm{PE}$, asymptotic convergence when $\Delta \notin \mathcal{L}_{2}$, and boundedness of the estimate $\hat{\theta}$ when $\Delta \in \mathcal{L}_{2}$. It is of interest to study the convergence conditions in the context of the DREM procedure (assuming the noise-free scenario) of the LSQ (Least Squares) estimator. 
For $i \in \bar{n}$, the LSQ estimator is given by (see [2])

$$
\begin{aligned}
& \dot{\hat{\theta}}_{i}(t)=\gamma_{i} \Delta(t) p_{i}(t)\left(\mathcal{Y}_{i}(t)-\Delta(t) \hat{\theta}_{i}(t)\right), \\
& \dot{p}_{i}(t)=\gamma_{i}\left(\lambda_{i} p_{i}(t)-p_{i}^{2}(t) \Delta^{2}(t)\right),
\end{aligned}
$$

where $p_{i}(0)>0$ and $\gamma_{i}>0$ are the design parameters, and $\lambda_{i} \geq 0$ is the forgetting factor. The error dynamics under the LSQ estimator is given by

$$
\dot{\tilde{\theta}}_{i}(t)=-\gamma_{i} \Delta^{2}(t) p_{i}(t) \tilde{\theta}_{i}(t) .
$$

The properties of the LSQ estimator are well-studied, and the possible unboundedness of $p_{i}$ for $\lambda_{i}>0$ and $\Delta$ not being $\mathrm{PE}$ is known, see for example, [3]. However, since the DREM procedure renders the LRE (1) to the set of scalar equations (4), we consider the scalar realization of the LSQ estimator, and thus we can analyze the exact solution of $p_{i}$. This provides a conclusion for the case when $\Delta \notin \mathrm{PE}$ but $\Delta \notin \mathcal{L}_{2}$, which is an important situation for the DREM procedure and which is not typically addressed for the LSQ estimators.

Remark 3: It is worth noting that for a bounded scalar signal $\Delta$, if $\Delta \in \mathrm{PE}$ then it implies that $\Delta \notin \mathcal{L}_{2}$. However, $\Delta \notin \mathcal{L}_{2}$ does not necessarily implies that $\Delta \in \mathrm{PE}$ : that can be illustrated by $\Delta(t)=\frac{1}{\sqrt{t+1}}, \forall t$.

Properties of the LSQ estimator in the context of the DREM procedure are given in the following proposition.

Proposition 2: Let $i \in \bar{n}$. Consider the estimation algorithm (11), (12) with $p_{i}(0)>0$ and $\gamma_{i}>0$.

(i) If $\lambda_{i}=0$ (LSQ estimator without forgetting) then

1) if $\Delta \in \mathcal{L}_{2}$ then for all nonzero $\tilde{\theta}_{i}(0)$ the signal $\tilde{\theta}_{i}$ does not converge to zero;

2) if $\Delta \notin \mathcal{L}_{2}$ then $\tilde{\theta}_{i}$ is monotonic and converges to zero asymptotically;

3) if $\Delta$ is PE, it does not imply exponential convergence.

(ii) If $\lambda_{i}>0$ (LSQ estimator with forgetting) then

1) $p_{i}$ is bound from below as

2) if $\Delta \in \mathcal{L}_{2}$ or

$$
\Delta \notin \mathcal{L}_{2} \text { and } \Delta \rightarrow 0,
$$

then the estimator is unstable and $p_{i}$ tends to infinity;

$3)$ if $\Delta$ is PE then $p_{i}$ is bounded, $\tilde{\theta}_{i}$ is monotonic and converges to zero exponentially fast.

Proof: Part 1. Consider first the case $\lambda_{i}=0$. We obtain

$$
\dot{p}_{i}(t)=-\gamma_{i} p_{i}^{2}(t) \Delta^{2}(t)
$$

giving

$$
p_{i}(t)=\frac{1}{c_{1}+\gamma_{i} \int_{0}^{t} \Delta^{2}(s) d s},
$$

where $c_{1}=\frac{1}{p_{i}(0)}$. Obviously, for $\Delta \notin \mathcal{L}_{2}$ we have $p_{i} \rightarrow 0$, as it is expected for an LSQ estimator without forgetting. The error dynamics can be now written as

$$
\dot{\tilde{\theta}}(t)=-\beta(t) \tilde{\theta},
$$

where

$$
\beta(t)=\frac{\gamma_{i} \Delta^{2}(t)}{c_{1}+\gamma_{i} \int_{0}^{t} \Delta^{2}(s) d s}=\frac{\Delta^{2}(t)}{c_{2}+\int_{0}^{t} \Delta^{2}(s) d s},
$$

and $c_{2}=\frac{c_{1}}{\gamma_{i}}$. For a bounded function $\Delta$, the LTV system (14) has the unique solution

$$
\tilde{\theta}_{i}(t)=\frac{c_{3}}{c_{2}+\int_{0}^{t} \Delta^{2}(s) d s},
$$

where $c_{3}=\tilde{\theta}(0) c_{2}$. Indeed, taking the time derivative of (16) we obtain

$$
\begin{aligned}
\dot{\tilde{\theta}}(t) & =-\frac{c_{3} \Delta^{2}(t)}{\left(c_{2}+\int_{0}^{t} \Delta^{2}(s) d s\right)^{2}} \\
& =-\frac{\Delta^{2}(t)}{c_{2}+\int_{0}^{t} \Delta^{2}(s) d s} \cdot \frac{c_{3}}{c_{2}+\int_{0}^{t} \Delta^{2}(s) d s}=-\beta(t) \tilde{\theta} .
\end{aligned}
$$

From (16), we observe that $\tilde{\theta}_{i}$ does not converge to zero if $\Delta \in \mathcal{L}_{2}$ and $c_{3} \neq 0$. On the other hand, for $\Delta \notin \mathcal{L}_{2}$ it follows from (16) that $\tilde{\theta}$ converges to zero asymptotically. Moreover, since the function $\beta$ defined in (15) is nonnegative, the convergence is monotonic. Finally, notice that a PE $\Delta$ implies that $\beta$ in (14) converges to zero that prevents $\tilde{\theta}$ to converge exponentially; for the exponential convergence the PE property of $\beta$ is required.

Part 2. Consider now the case $\lambda_{i}>0$. The nonlinear ODE (12) has the solution

$$
p_{i}(t)=\frac{e^{\lambda_{i} \gamma_{i} t} p_{i}(0)}{1+p_{i}(0) \gamma_{i} \int_{0}^{t} e^{\lambda_{i} \gamma_{i} s} \Delta^{2}(s) d s} .
$$

The rest of proof is performed in three steps. First, we show that for all bounded $\Delta, p_{i}$ is bounded from below by a positive constant. Second, we show that $p_{i}$ is bounded from above since $\Delta$ being PE implies exponential convergence of $\tilde{\theta}$. Finally, we show that $p_{i}$ tends to infinity if $\Delta \in \mathcal{L}_{2}$ or if $\Delta$ tends to zero.

Step 1. Consider the inverse function

$$
\frac{1}{p_{i}(t)}=\frac{1}{p_{i}(0)} e^{-\lambda_{i} \gamma_{i} t}+\gamma_{i} z(t),
$$

where

$$
z(t)=e^{-\lambda_{i} \gamma_{i} t} \int_{0}^{t} e^{\lambda_{i} \gamma_{i} s} \Delta^{2}(s) d s .
$$

Recalling that $\Delta$ is bounded, say $\Delta^{2}(t) \leq \bar{\Delta}$, then the function $z$ is bounded as

$$
z(t) \leq \bar{\Delta} e^{-\lambda_{i} \gamma_{i} t} \int_{0}^{t} e^{\lambda_{i} \gamma_{i} s} d s=\frac{\bar{\Delta}}{\lambda_{i} \gamma_{i}}\left(1-e^{-\lambda_{i} \gamma_{i} t}\right) \leq \frac{\bar{\Delta}}{\lambda_{i} \gamma_{i}} .
$$

It follows that $\frac{1}{p_{i}(t)} \leq \frac{1}{p_{i}(0)}+\frac{\bar{\Delta}}{\lambda_{i}}$, and $p_{i}$ is bounded from below as

$$
p_{i}(t) \geq \frac{p_{i}(0) \lambda_{i}}{\lambda_{i}+\bar{\Delta} p_{i}(0)}=p_{m} .
$$

Step 2. Assume that $\Delta$ is $(T, \mu)$-PE. Then for $t \geq T$ the function $z$ is bounded from below as

$$
\begin{aligned}
z(t) & \geq e^{-\lambda_{i} \gamma_{i} t} \int_{t-T}^{t} e^{\lambda_{i} \gamma_{i} s} \Delta^{2}(s) d s \\
& \geq e^{-\lambda_{i} \gamma_{i} t} e^{\lambda_{i} \gamma_{i}(t-T)} \int_{t-T}^{t} \Delta^{2}(s) d s \geq \mu e^{-\lambda_{i} \gamma_{i} T} .
\end{aligned}
$$


Then we have the following bounds:

$$
\begin{aligned}
& \frac{1}{p_{i}(t)} \geq \frac{1}{p_{i}(0)} e^{-\lambda_{i} \gamma_{i} T} \quad \text { for } 0 \leq t \leq T, \\
& \frac{1}{p_{i}(t)} \geq \gamma_{i} z(t) \geq \gamma_{i} \mu e^{-\lambda_{i} \gamma_{i} T} \quad \text { for } t>T \text {. }
\end{aligned}
$$

Hence the function $p_{i}$ is bounded by

$$
p_{i}(t) \leq e^{\lambda_{i} \gamma_{i} T} \max \left(p_{i}(0), \frac{1}{\mu \gamma_{i}}\right)=p_{M} .
$$

From (18) and (19) it follows that if $\Delta$ is PE, then the signal $t \mapsto \sqrt{p_{i}(t)} \Delta(t)$ is bounded and PE as well. Therefore the exponential convergence of $\tilde{\theta}$ follows from (13). Moreover, the convergence is monotonic since $p_{i}(t) \Delta^{2}(t) \geq 0$, $\forall t \geq 0$.

Step 3. Assume now that $\Delta \in \mathcal{L}_{2}$. Rewriting $z$ as

$$
z(t)=\int_{0}^{t} e^{-\lambda_{i} \gamma_{i}(t-s)} \Delta^{2}(s) d s
$$

it can be noted that $z$ is the solution of the differential equation

$$
\dot{z}(t)+\lambda_{i} \gamma_{i} z(t)=\Delta^{2}(t), z(0)=0 .
$$

Note that $\Delta \in \mathcal{L}_{2}$ implies $\Delta^{2} \in \mathcal{L}_{1}$. It is known that the considered stable first order linear time-invariant (LTI) system (20) has a finite $\mathcal{L}_{1}$ gain, thus $z \in \mathcal{L}_{1}$. Noting also that $\dot{z}$ is bounded and applying Barbalat's lemma we conclude $z \rightarrow 0$. Then from (17) it follows that $p_{i}$ tends to infinity and the estimator (11), (12) is unstable.

For the case when $\Delta \notin \mathcal{L}_{2}$ but $\Delta \rightarrow 0$, we note that the LTI system (20) is exponentially stable, therefore for $\Delta^{2} \rightarrow 0$ we have $z \rightarrow 0$ and $p_{i}$ tends to infinity.

Remark 4: Proposition 2 shows that (within the context of the DREM procedure) the use of the LSQ estimator (11), (12) does not provide any improvement in the convergence conditions with respect to the gradient estimator (6) and can yield unboundedness of the trajectories when $\Delta \rightarrow 0$.

\section{Simulations}

\section{A. Preservation of the excitation}

To illustrate the results obtained in Section III we consider the problem of parameter estimation for a sinusoidal signal with a known frequency. Consider $y(t)=B+A \sin (t+$ $\psi)$, where $B, A>0$, and $\psi \in[-\pi, \pi)$ are the unknown parameters. This signal can be rewritten as the LRE (1) with

$$
\begin{aligned}
\phi(t) & =\left[\begin{array}{lll}
1 & \sin (t) & \cos (t)
\end{array}\right]^{\top}, \\
\theta & =\left[\begin{array}{lll}
B & A \cos (\psi) & A \sin (\psi)
\end{array}\right]^{\top},
\end{aligned}
$$

and $w \equiv 0$. For simulations, we set $B=2, A=\sqrt{2}$, and $\psi=-\frac{1}{4} \pi$ providing $\theta=\left[\begin{array}{lll}2 & 1 & -1\end{array}\right]^{\top}$.

It is straightforward to verify that the regressor $\phi$ is $(2 \pi, \pi)$-PE, i.e., for all $t \geq 0$

$$
\int_{t}^{t+2 \pi} \phi(s) \phi^{\top}(s) d s \geq \pi I_{3}
$$

First we apply the standard gradient algorithm (2) with $\Gamma=I_{3}$ and $\hat{\theta}(0)=0$; the simulation results are depicted

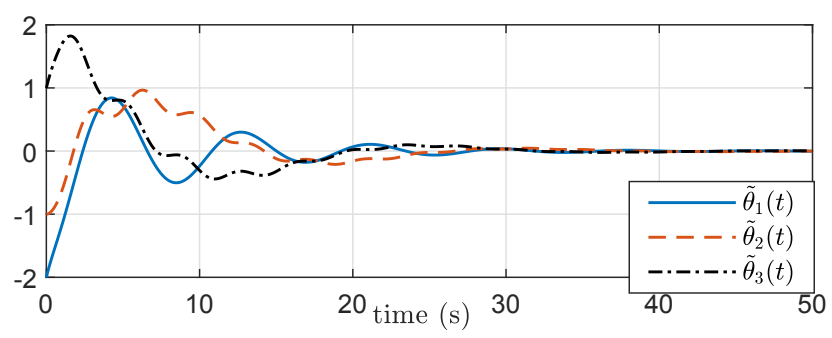

(a) The standard gradient algorithm (2).

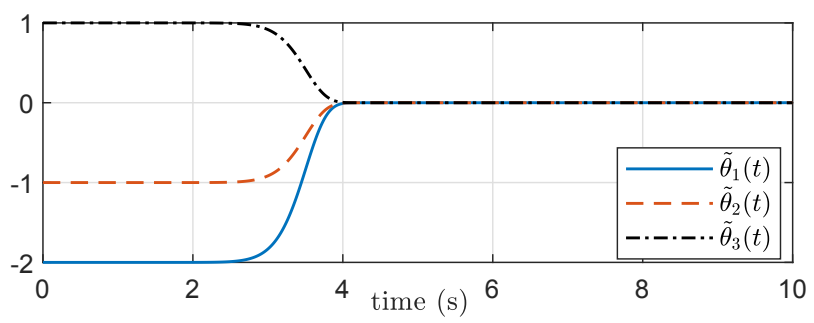

(b) The DREM procedure (7), (8) and the gradient estimator (6).

Fig. 1: The estimation error $\tilde{\theta}$.

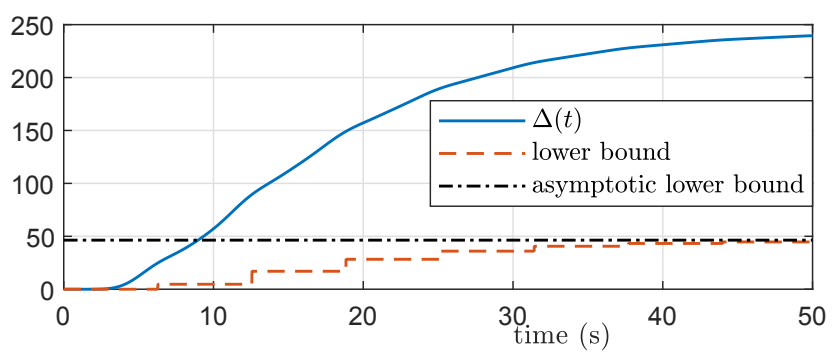

Fig. 2: The new regressor $\Delta$, the lower bound (9), and the asymptotic lower bound (10).

in Fig. 1a. Since $\phi$ is PE, the estimation error $\tilde{\theta}$ converges to zero; however, the transients exhibit oscillations. Then we apply the dynamic regressor extension (7), (8), where the only tuning parameter is chosen as $a=0.1$. The estimation error transient $\tilde{\theta}$ for the DREM procedure with the gradient estimator (6) with $\gamma_{i}=1, i=1,2,3$, are depicted in Fig. 1b and illustrate performance improvement with respect to the standard gradient estimator; note the difference in the time scale and the monotonicity of the transients.

The new regressor $\Delta$ computed as (5) is depicted in Fig. 2 with the lower bound (9) and with the asymptotic lower bound (10).

\section{B. DREM with the LSQ estimator}

To illustrate Proposition 2 and possible unboundedness of the LSQ estimator, we consider two regression models of the form (1) with the regressors $\phi_{1} \in \mathrm{PE}$ and $\phi_{2} \notin \mathrm{PE}$ where

$$
\phi_{1}(t)=\left[\begin{array}{ll}
1 & \sin (t)
\end{array}\right]^{\top}, \phi_{2}(t)=\left[\begin{array}{ll}
1 & \frac{\sin (t)}{\sqrt{t+2 \pi}}
\end{array}\right]^{\top}, \forall t \geq 0
$$

and the vector of unknown parameters $\theta=\left[\begin{array}{ll}2 & 1\end{array}\right]^{\top}$.

To apply the DREM procedure, we consider time-delay 


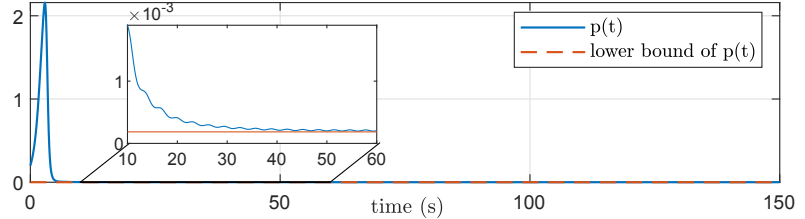

(a) $\Delta_{1} \in \mathrm{PE}$.

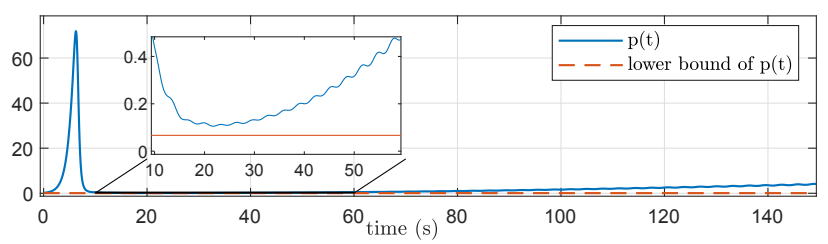

(b) $\Delta_{2} \notin \mathcal{L}_{2}$.

Fig. 3: The signal $p$ and the lower bound (18).

operators and define

$$
\Phi_{i}(t)=\left[\begin{array}{ll}
\phi_{i}(t) & \phi_{i}(t-\pi)
\end{array}\right]^{\top}, \Delta_{i}(t)=\operatorname{det} \Phi_{i}(t),
$$

where $i=1,2$. It is straightforward to show that $\Delta_{1} \in \mathrm{PE}$ and $\Delta_{2}(t) \rightarrow 0$ as $t \rightarrow \infty$.

The LSQ estimator (11)-(12) with $\lambda_{1}=0.5$ and $\gamma_{1}=$ 0.5 is applied for both models. For the persistently excited regressor $\Delta_{1}$ the corresponding signal $p$ is bounded and tends to the lower bound (18) as shown in Fig. 3a. The behavior of the LSQ estimator for the decaying regressor $\Delta_{2}$ is depicted in Fig. $3 \mathrm{~b}$ and illustrates that the signal $p$ is not bounded and tends to infinity.

\section{CONCLUSION}

The recently proposed DREM procedure provides significant performance improvement in linear regression parameter estimation, where the main degree of freedom is the choice of the dynamic extension operator. In this paper, we have studied a special choice of this operator as the Kreisselmeier's regressor extension (7), (8) that ensures the preservation of the persistence of excitation property. In particular, we proved that the determinant of the extended matrix is persistently exciting if the original regressor is. This result alleviates the main design question of the DREM procedure.

We have also considered the use of the LSQ estimator within the DREM procedure. It is shown that for the resulting scalar linear regression equations, the LSQ estimator does not provide any advantage in the convergence properties and it can be unbounded if the regressor $\Delta$ decays.

Our further researches are oriented towards the extension of Theorem 1 for the case when $\phi \notin \mathrm{PE}$. We expect that eigenvalue conditions similar to the ones reported in [19] can be formulated. We also intend to study the propagation of (the non-uniform in time) interval excitation.

\section{REFERENCES}

[1] L. Ljung, System Identification: Theory for User. New Jersey: Prentice Hall, 1987.
[2] S. Sastry and M. Bodson, Adaptive control: Stability, convergence, and robustness. New Jersey: Prentice-Hall, 1989.

[3] P. A. Ioannou and J. Sun, Robust Adaptive Control. New Jersey: Prentice-Hall, 1996.

[4] M. M'Saad, R. Ortega, and I. Landau, "Adaptive controllers for discrete-time systems with arbitrary zeros: An overview," Automatica, vol. 21, no. 4, pp. 413-423, jul 1985.

[5] G. C. Goodwin and K. S. Sin, Adaptive filtering prediction and control. Courier Corporation, 2014.

[6] F. Lewis, D. Vrabie, and K. Vamvoudakis, "Reinforcement Learning and Feedback Control: Using Natural Decision Methods to Design Optimal Adaptive Controllers," IEEE Control Systems, vol. 32, no. 6 , pp. 76-105, 2012.

[7] D. Efimov and A. Fradkov, "Design of impulsive adaptive observers for improvement of persistency of excitation," International Journal of Adaptive Control and Signal Processing, vol. 29, no. 6, pp. 765-782, jun 2015.

[8] S. Aranovskiy, A. Bobtsov, R. Ortega, and A. Pyrkin, "Performance Enhancement of Parameter Estimators via Dynamic Regressor Extension and Mixing*," IEEE Transactions on Automatic Control, vol. 62, no. 7, pp. 3546-3550, jul 2017.

[9] R. Ortega, L. Praly, S. Aranovskiy, B. Yi, and W. Zhang, "On dynamic regressor extension and mixing parameter estimators: Two Luenberger observers interpretations," Automatica, vol. 95, pp. 548-551, sep 2018

[10] D. N. Gerasimov, R. Ortega, and V. O. Nikiforov, "Relaxing the highfrequency gain sign assumption in direct model reference adaptive control," European Journal of Control, vol. 43, pp. 12-19, sep 2018.

[11] A. A. Vedyakov, A. O. Vediakova, A. A. Bobtsov, A. A. Pyrkin, and S. V. Aranovskiy, "A globally convergent frequency estimator of a sinusoidal signal with a time-varying amplitude," European Journal of Control, vol. 38, pp. 32-38, nov 2017.

[12] A. Bobtsov, D. Bazylev, A. Pyrkin, S. Aranovskiy, and R. Ortega, "A robust nonlinear position observer for synchronous motors with relaxed excitation conditions," International Journal of Control, vol. 90 , no. 4, pp. 813-824, apr 2017.

[13] J. Schiffer, P. Aristidou, and R. Ortega, "Online Estimation of Power System Inertia Using Dynamic Regressor Extension and Mixing," IEEE Transactions on Power Systems, pp. 1-1, 2019.

[14] A. Pyrkin, F. Mancilla-David, R. Ortega, A. Bobtsov, and S. Aranovskiy, "Identification of photovoltaic arrays' maximum power extraction point via dynamic regressor extension and mixing," International Journal of Adaptive Control and Signal Processing, vol. 31 , no. 9, pp. 1337-1349, sep 2017.

[15] R. Ortega, S. Aranovskiy, A. Pyrkin, A. Astolfi, and A. Bobtsov, "New Results on Parameter Estimation via Dynamic Regressor Extension and Mixing: Continuous and Discrete-time Cases," Submitted to IEEE Transactions on Automatic Control, 2019.

[16] S. Aranovskiy, A. Belov, R. Ortega, N. Barabanov, and A. Bobtsov, "Parameter identification of linear time-invariant systems using dynamic regressor extension and mixing," International Journal of Adaptive Control and Signal Processing, vol. 33, no. 6, pp. 10161030, jun 2019

[17] G. Kreisselmeier, "Adaptive observers with exponential rate of convergence," IEEE Transactions on Automatic Control, vol. 22, no. 1, pp. 2-8, feb 1977.

[18] D. N. Gerasimov, M. E. Belyaev, and V. O. Nikiforov, "Performance improvement of discrete mrac by dynamic and memory regressor extension," in 2019 18th European Control Conference (ECC). IEEE, 2019, pp. 2950-2956.

[19] N. Barabanov and R. Ortega, "On global asymptotic stability of $\dot{x}=$ $-\phi(t) \phi^{\top}(t) x$ with $\phi(t)$ not persistently exciting," Systems \& Control Letters, vol. 109, pp. 24-29, nov 2017.

[20] J. Wang, D. Efimov, and A. Bobtsov, "On robust parameter estimation in finite-time without persistence of excitation," IEEE Transactions on Automatic Control, vol. 65, no. 4, pp. -, to appear in 2020, avaliable online.

[21] N. Cho, H.-S. Shin, Y. Kim, and A. Tsourdos, "Composite Model Reference Adaptive Control with Parameter Convergence Under Finite Excitation," IEEE Transactions on Automatic Control, vol. 63, no. 3 , pp. 811-818, mar 2018.

[22] M. Noack, J. G. Rueda-Escobedo, J. Reger, and J. A. Moreno, "Fixedtime parameter estimation in polynomial systems through modulating functions," in 2016 IEEE 55th Conference on Decision and Control (CDC). IEEE, 2016, pp. 2067-2072. 